\title{
Magnetic resonance imaging of traumatic and non-traumatic brachial plexopathies
}

Yiru Lorna $\underline{\text { Fan }}^{1}$, MBChB, FRCR, Mohamad Isham Bin $\underline{\text { Othman }}{ }^{2}$, MBBS, FRCR, Niraj Dubey ${ }^{2}$, MBBS, FRCR, Wilfred $C G$ Peh$^{2}$, FRCP, FRCR

\begin{abstract}
Adult-onset brachial plexopathy can be classified into traumatic and non-traumatic aetiologies. Traumatic brachial plexopathies can affect the pre- or postganglionic segments of the plexus. Non-traumatic brachial plexopathies may be due to neoplasia, radiotherapy, thoracic outlet syndrome and idiopathic neuralgic amyotrophy. Conventional magnetic resonance imaging (MRI) is useful to localise the area of injury or disease, and identify the likely cause. This review discusses some of the common causes of adult-onset brachial plexopathy and their imaging features on MRI. We also present a series of cases to illustrate some of these causes and their MRI findings.
\end{abstract}

Keywords: anatomy, brachial plexus, magnetic resonance imaging, neuropathies, trauma

\section{INTRODUCTION}

Brachial plexopathies are conditions affecting the brachial plexus, the cluster of nerves that innervate the upper limb. Disease or disruption of the brachial plexus can severely affect the function of the upper limb musculature and sensation. The causes of brachial plexopathy in adults can be broadly divided into traumatic and non-traumatic aetiologies. Modalities used for brachial plexopathy imaging include standard myelography, computed tomography myelography, conventional magnetic resonance imaging (MRI) and MR myelography. Of these techniques, MRI is non-invasive, useful in providing detailed anatomic information and may provide accurate localisation of the pathology. We present a series of cases of brachial plexopathy in adults over a period of five years. These help to illustrate some of the common causes of adult-onset traumatic and non-traumatic brachial plexopathy, and their relevant MRI features.

\section{ANATOMY OF THE BRACHIAL PLEXUS}

The brachial plexus is formed by the nerve roots originating from the C5, C6, C7, C8 and T1 levels. The nerve roots are divided into pre- and postganglionic sections, with the dorsal root ganglion as the point of reference. The brachial plexus itself is formed by the ventral rami of the postganglionic sections of the nerve roots. It is subdivided into the following portions, from proximal to distal: five roots, three trunks, six divisions, three cords and five terminal nerves. The $\mathrm{C} 5$ and $\mathrm{C} 6$ nerve roots unite to form the upper trunk; the $\mathrm{C} 7$ nerve root forms the middle trunk; and the $\mathrm{C} 8$ and $\mathrm{T} 1$ nerve roots form the lower trunk. Each of these three trunks then divide into anterior and posterior divisions, i.e. six divisions in all. The trunks and divisions are found superior to the clavicle.

The three posterior divisions unite to form the posterior cord. The anterior divisions of the upper and middle trunk unite to form the lateral cord. The remaining anterior division of the lower trunk continues as the medial cord. The cords are found inferior to the clavicle. The posterior cord continues on to form the radial and axillary nerves. The lateral cord continues on as the musculocutaneous nerve, while the medial cord continues on as the ulnar nerve. The lateral and medial cords also supply fibres that unite to form the median nerve.

\section{MAGNETIC RESONANCE IMAGING OF THE BRACHIAL PLEXUS}

MRI of the peripheral nerves (MR neurography) has been increasingly used in recent years, due to advancements in MRI techniques. It is an excellent modality for obtaining detailed anatomic information on the brachial plexus (Fig. 1) and to accurately localise any pathology. MRI of the brachial plexus can be performed on both 1.5T and 3T MR scanners, and uses combinations of two-dimensional (2D) and three-dimensional (3D) techniques. 2D short tau inversion recovery (STIR) sequences are frequently part of imaging protocols on 1.5T scanners. However, they have certain disadvantages including suboptimal spatial resolution, occurrence of pulsation artefacts, and poor discrimination between nerves and blood vessels. ${ }^{(1)} \mathrm{A}$ newer technique is 2D T2-weighted spectrally adiabatic inversion recovery (SPAIR; Siemens Healthineers, Erlangen, Germany), which is superior to STIR on 3T scanners as it has a better signal-to-noise ratio.(1) SPAIR also demonstrates more uniform fat suppression than STIR in larger fields of view, such as the brachial plexus. ${ }^{(2)}$ 3D STIR sampling perfection with application-optimised contrasts using different flip angle evolution (SPACE; Siemens Healthineers, Erlangen, Germany) sequences can be performed on both 1.5T and 3T scanners. They allow multiplanar reconstructions along the longitudinal axis of a nerve, which can better demonstrate any pathological changes in a nerve compared to $2 \mathrm{D}$ techniques. ${ }^{(1)}$

In our institution, we currently use a Siemens 1.5T MAGNETOM $®$ Avanto system (Siemens Healthineers, Erlangen, Germany), with head, neck and spine matrix coils, and a body 

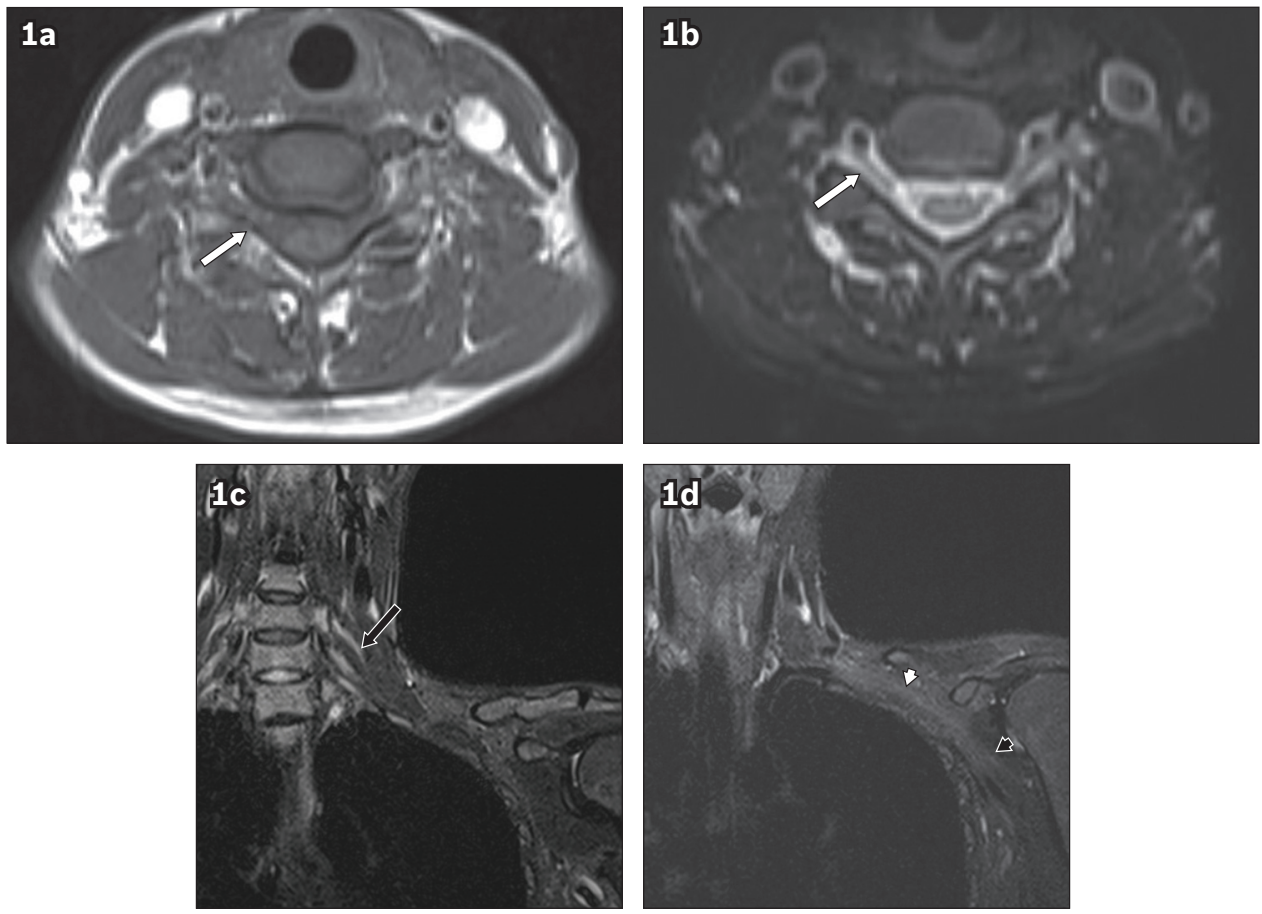

Fig. 1 Axial (a) T1-W (b) T2-W MR images of the brachial plexus show the nerve roots (white arrows) exiting the neural foramen. (c \& d) Coronal short tau inversion recovery images show the trunks (black arrow in c), cords (white arrowhead in d) and terminal branches (black arrowhead in d).

Table I. Magnetic resonance protocol for evaluation of the brachial plexus.

\begin{tabular}{|c|c|c|c|c|c|}
\hline Sequence & Field of view $(\mathrm{mm})$ & $\begin{array}{c}\text { Slice thickness/ } \\
\text { interslice gap }(\mathrm{mm})\end{array}$ & Resolution & TR (ms) & TE (ms) \\
\hline Axial T1-weighted TSE & 350 & $3.5 / 0.7$ & $384 \times 268$ & 441 & 10 \\
\hline Axial T2-weighted TSE (fat-suppressed) & 350 & $3.5 / 0.7$ & $320 \times 224$ & 3,460 & 74 \\
\hline Axial T2-weighted haeme & 280 & $3.5 / 0.7$ & $256 \times 204$ & 1,460 & 26 \\
\hline Coronal T1-weighted TSE & 350 & $3.5 / 0.3$ & $384 \times 268$ & 531 & 10 \\
\hline Coronal STIR & 350 & $3.5 / 0.3$ & $320 \times 224$ & 3,610 & 72 \\
\hline Coronal STIR SPACE & 350 & 1.2 & $320 \times 320$ & 2,500 & 295 \\
\hline Oblique sagittal T1-weighted TSE & 260 & $4.5 / 0.4$ & $320 \times 224$ & 613 & 9 \\
\hline Oblique sagittal STIR & 260 & $4.5 / 0.4$ & $256 \times 204$ & 3,540 & 75 \\
\hline
\end{tabular}

SPACE: sampling perfection with application-optimised contrasts using different flip angle evolution; STIR: short tau inversion recovery; TE: echo time; TR: repetition time; TSE: turbo spin-echo

array coil. Imaging is performed in the axial, coronal and oblique sagittal planes (Table I). Axial images parallel to the disc spaces, coronal images parallel to the vertebrae and shoulders, and oblique sagittal images perpendicular to the brachial plexus are obtained. Axial T1-weighted sequence using turbo spin-echo (TSE) imaging and T2-weighted sequence using TSE with fat suppression are performed. In cases of trauma, an additional T2-weighted sequence for susceptibility (haeme) is performed. Coronal T1-weighted sequence with TSE, STIR sequence and STIR SPACE sequence, and oblique sagittal T1-weighted sequence with TSE and STIR sequence are also performed for all imaging of the brachial plexus. Intravenous gadolinium-based contrast is not routinely used in imaging of the brachial plexus. Its main utility is in evaluating possible neoplastic and inflammatory processes.

\section{TRAUMATIC BRACHIAL PLEXOPATHY}

Injuries sustained in road traffic accidents, particularly those involving motorcyclists, are a major contributing cause of traumatic brachial plexopathy. ${ }^{(3)}$ Other forms of trauma include falls from a height, an impact on the shoulder from falling heavy objects, and sports injuries. Depending on the nature and aetiology of the trauma, different sections of the brachial plexus may be affected.

A classification of the severity of nerve injuries was proposed by Seddon, ${ }^{(4)}$ using the terms neuropraxia, axonotmesis and neurotmesis (from least to most severe). The least severe grade, neuropraxia, involves damage to the myelin sheath but not the axon. Wallerian degeneration does not occur and the conduction defects are temporary, with spontaneous and complete recovery. In the next grade, axonotmesis, there is damage to the axon as well as Wallerian degeneration. However, the surrounding connective tissues are intact. Axonal regeneration leads to slower recovery. In the most severe grade, neurotmesis, there is complete disruption of the myelin, axon as well as the surrounding connective tissues. Wallerian degeneration occurs and spontaneous recovery is unlikely; 

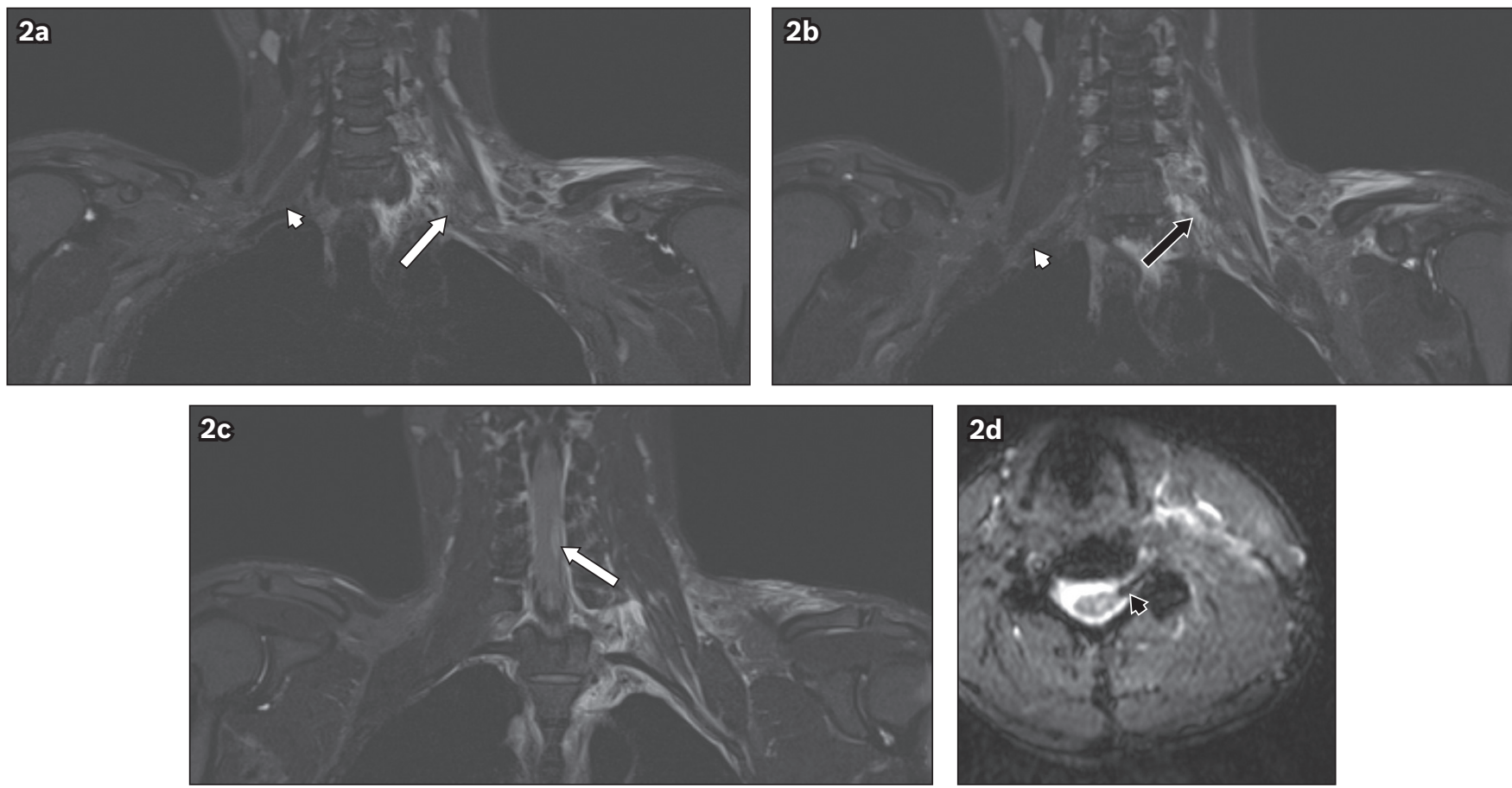

Fig. 2 A 28-year-old male motorcyclist presented after a collision with a lorry. He was found to have reduced power in the left upper limb in the C5 to T1 muscle groups, with reduced sensation from the $\mathrm{C} 5$ to $\mathrm{C} 7$ dermatomes. On MRI, the left $\mathrm{C} 6$ to $\mathrm{T} 1$ nerve roots were discontinuous. (a \& b) Coronal short tau inversion recovery (STIR) sequence images show injuries to the left C6 (white arrow in a) and C7 (black arrow in b) nerve roots. In comparison, the right-sided nerve roots show continuity (white arrowheads in a \& b). This is consistent with traumatic disruption of the left brachial plexus. Hyperintense signal (white arrow in c) on the coronal STIR image in the left lateral spinal cord at the level of C5 suggests oedema from preganglionic injury. The susceptibility artefact on the haeme-sensitive sequence due to subacute blood products at the expected location of the left C6 nerve root in the epidural space likely represents a root avulsion injury (black arrowhead in d). T2-hyperintense signal in the left supraclavicular region is in keeping with contusions.

therefore, surgical repair is typically necessary. Neuromas can develop at the stumps of transected nerves that have not regenerated.

\section{Root avulsion injury and preganglionic injury of the brachial plexus}

Root avulsion injury of the brachial plexus occurs when the attachment of the nerve root to the spinal cord is disrupted. It includes preganglionic injuries to the nerve roots, but can present together with injuries to other distal sections of the brachial plexus. It typically arises from high-energy impacts such as those that occur in road traffic accidents, usually causing traction of the nerve roots. The $\mathrm{C} 7$ to $\mathrm{T} 1$ nerve roots are most commonly avulsed in a traction injury. ${ }^{(5)}$ Traction can also lead to the epidural sleeve being pulled away from the spinal cord, leading to the formation of a pseudomeningocoele. Preganglionic injuries involve neurons that do not regenerate, leading to a limited potential for recovery of function in the affected nerves. They are usually treated with nerve transfers, in which nerves from other sites are harvested and used to connect to the nerve stumps. ${ }^{(6)}$

MRI features of root avulsion and preganglionic injuries include: $^{(6)}$ (a) oedema of the adjacent spinal cord, with T2hyperintense signal change (Fig. 2); (b) haemorrhage in the nerve root or adjacent spinal cord, with susceptibility artefacts on haeme-sensitive sequences (Figs. 2 and 3); and (c) the presence of pseudomeningocoeles, with well-defined T2-hyperintense collections of cerebrospinal fluid intensity
(Fig. 4). Enhancement of the paraspinal muscles with contrast administration, which reflects disruption of the nerve supply to the muscles, is another feature supporting the diagnosis of root avulsion injury.

\section{Postganglionic injuries of the brachial plexus}

Postganglionic injuries of the brachial plexus include rupture of the affected section of the neurons, as well as stretch injuries in which the continuity of the neurons is preserved, i.e. neuropraxia. They are also usually caused by high energy impacts causing traction of the nerve roots. Thus, postganglionic injuries can occur together with root avulsion injuries and preganglionic injuries. The majority of postganglionic injuries are found superior to the clavicle, affecting the roots and trunks. In particular, the C5 and C6 nerve roots, and the upper trunk are often involved in traction injuries. Postganglionic injuries inferior to the clavicle, affecting the terminal nerves, are less common. The axillary and musculocutaneous nerves are prone to traction injuries, as they are fixed during their course in the glenohumeral area. ${ }^{(5)}$

Postganglionic ruptures usually confer a better prognosis for functional recovery compared to preganglionic injuries, as the affected neurons have better potential for regeneration. These ruptures are usually treated with nerve grafting, in which the damaged section of the nerve is removed and the two remaining ends are joined together. ${ }^{\left({ }^{6}\right)}$ Stretch injuries without rupture tend to have better outcomes and are usually managed with conservative treatment and therapy. 

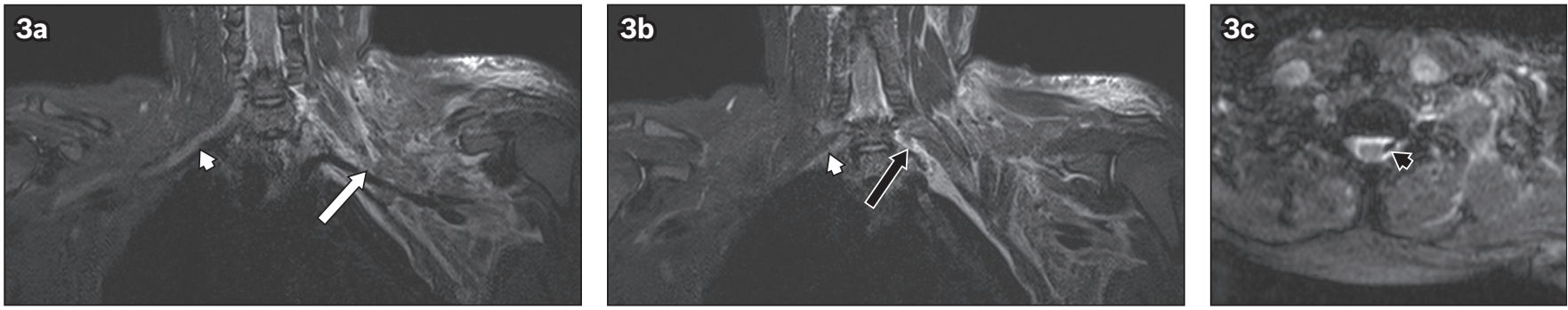

Fig. 3 A 32-year-old female motorcyclist was found by the side of the road following a road traffic accident. She sustained multiple injuries, and had absent power and sensation in her left upper limb. On MRI, the left C5 to T1 nerve roots were discontinuous. Coronal short tau inversion recovery sequence images show injuries to the left C7 (white arrow in a) and T1 (black arrow in b) nerve roots. In comparison, the right-sided nerve roots show continuity from their origins to the axilla (white arrowheads in a \& b). This is consistent with traumatic disruption of the left brachial plexus. The susceptibility artefact on the haeme-sensitive sequence, due to subacute blood products at the expected location of the left C7 nerve root in the epidural space, likely represents a root avulsion injury (black arrowhead in c). T2-hyperintense signal in the left supraclavicular and axillary regions is in keeping with contusions.
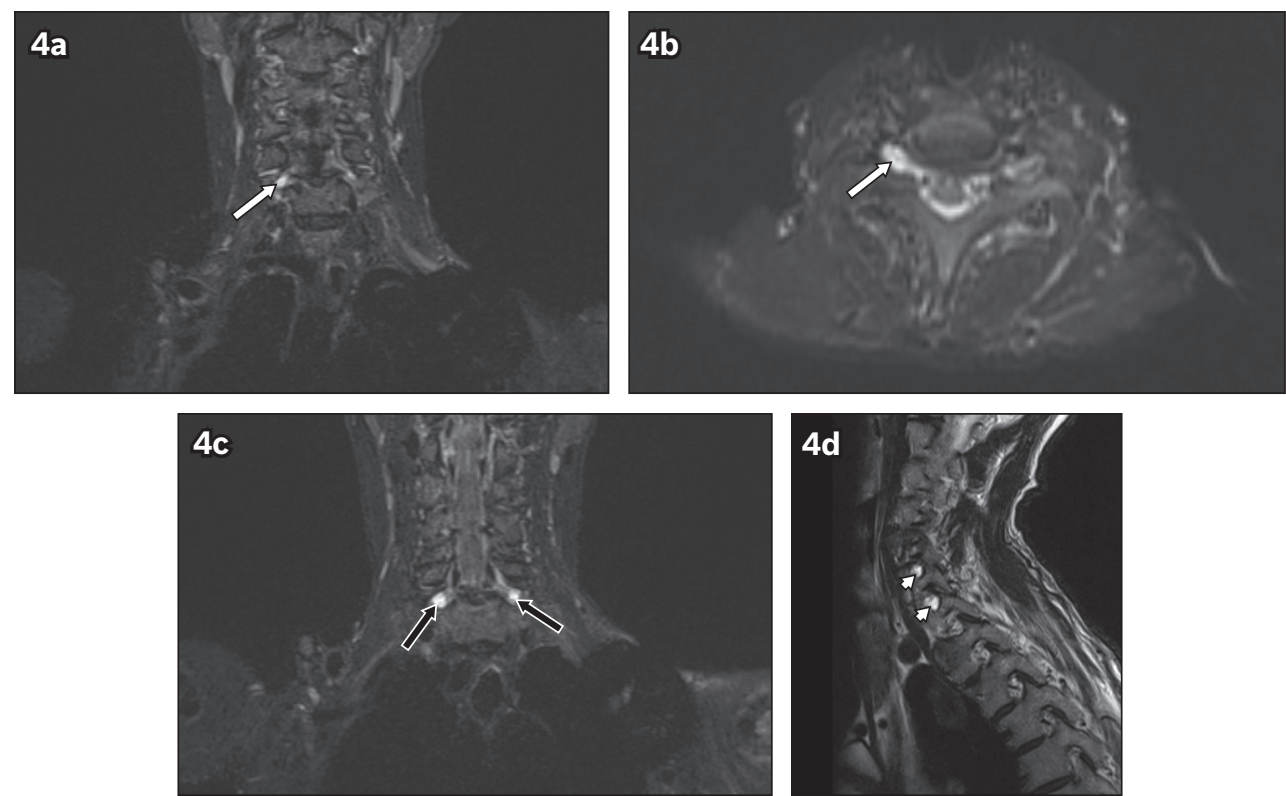

Fig. 4 A 54-year-old man fell from a height of $2 \mathrm{~m}$. He sustained fractures to the clavicle and scapula, and underwent internal fixation. The patient was noted to have residual signs of brachial plexus injury two months after the initial accident. On MRI, T2-hyperintense foci of cerebrospinal fluid signal intensity are seen at the neural foramina of the C6 to C8 nerve roots. No exiting nerve roots are evident within these foci. (a) Coronal and (b) axial short tau inversion recovery (STIR) images show the affected right C6 neural foramen (white arrows). (c) Coronal STIR sequence image shows the affected bilateral C7 neural foramina (black arrows). (d) Sagittal T2-W image shows the affected C7 and C8 neural foramina (arrowheads). These findings are compatible with post-traumatic pseudomeningocoeles.
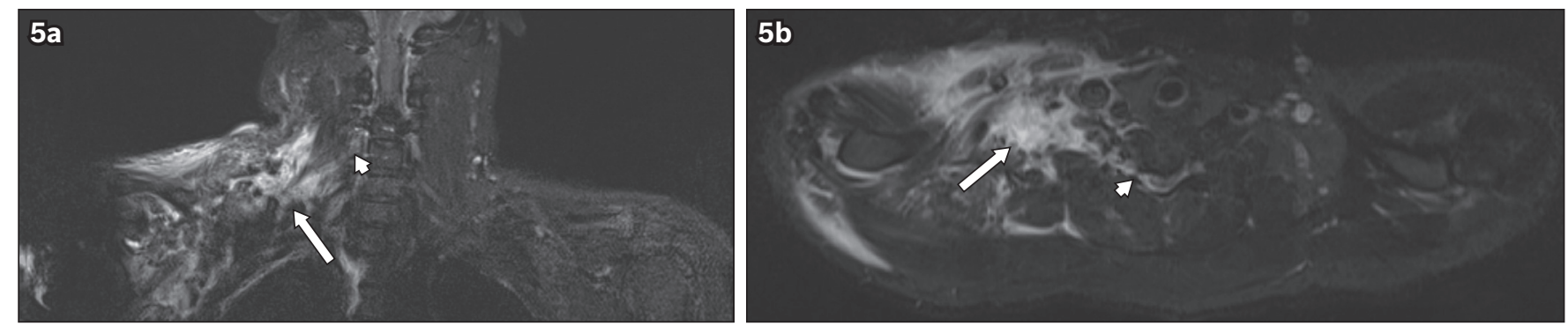

Fig. 5 A 31-year-old male motorcyclist was hit by a car. He sustained a fracture of the right humerus and underwent internal fixation. The patient also had loss of sensation and power in the right upper limb. MRI of the brachial plexus was performed. (a) Coronal and (b) axial short tau inversion recovery sequence images show the nerve roots of the right brachial plexus, which can be identified at their origins (arrowheads) but are indistinct in the supraclavicular region (arrows). The trunks are also indistinct. This is compatible with postganglionic traumatic disruption of the right brachial plexus. Extensive T2-hyperintense signal in the right supraclavicular region extending to the axilla is in keeping with contusions.

In postganglionic rupture, MRI shows that the nerve roots are identifiable at the origin at the spinal cord, but become indistinct more distally at the level of the rupture (Fig. 5). This is associated with retraction of the proximal segment. ${ }^{(7)}$ Other features include: (a) collections of fluid around the ruptured nerves, which are T2-hyperintense; (b) thickening of the nerves representing fibrosis when the injury is chronic; and (c) denervation of the supraspinatus and infraspinatus muscles, showing oedema in the acute phase and atrophy in the chronic phase. ${ }^{(8)}$ Neuropraxic postganglionic injury manifests as thickening and $\mathrm{T} 2$ hyperintensity of the affected nerves (Fig. 6). In contrast to postganglionic rupture, the continuity of the nerves is preserved. ${ }^{(7)}$ 


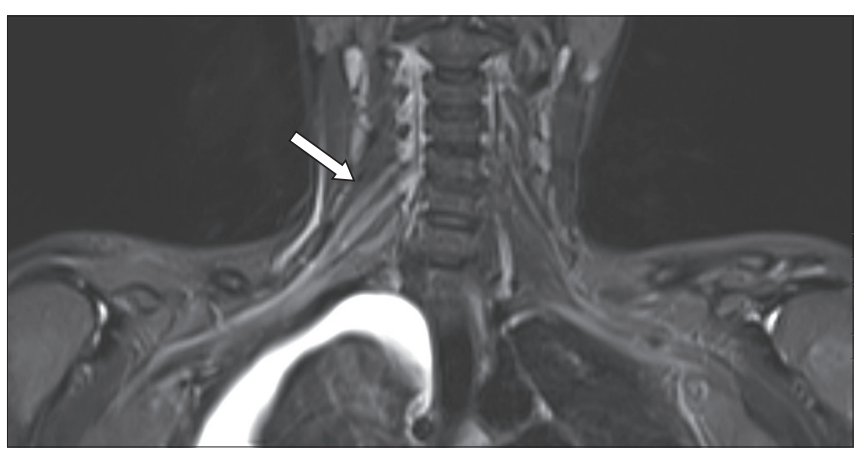

Fig. 6 A 26-year-old woman sustained multiple injuries following a fal from the fifth storey. She was noted to have residual right-sided upper limb weakness in the C5 and C6 dermatomes. On MRI, coronal short tau inversion recovery sequence image shows thickening and a hyperintense signal (arrow) in the right C5 to $\mathrm{C} 7$ nerve roots, extending to the trunks. No disruption of the brachial plexus is evident. This is compatible with traumatic neuropraxic postganglionic injury. A right pleural effusion is also noted.

\section{Stinger injury}

A stinger or burner injury is an injury to the brachial plexus caused by compression of the upper trunk, commonly affecting the $\mathrm{C} 5$ and $\mathrm{C} 6$ nerve roots. Its name refers to the sensation of pain experienced by the patient after the trauma. It is usually caused by direct forceful trauma to the neck or shoulder by a blunt object and is commonly seen in contact sports, e.g. American football and rugby; it can also result from heavy objects falling onto the neck or shoulder. The acute nature of the injury results in the patient presenting with pain in the supraclavicular and shoulder region, radiating down the left arm. The symptoms may be transient with complete recovery if only neuropraxia occurs; however, some injuries with more severe nerve damage may lead to chronic weakness, numbness or paraesthesia in the shoulder and arm. Its MRI features are similar to those of neuropraxic postganglionic injury: thickening and $\mathrm{T} 2$ hyperintensity of the affected nerves with no discontinuity of the nerves ${ }^{(7)}$ (Fig. 7).

\section{NON-TRAUMATIC BRACHIAL PLEXOPATHY}

Non-traumatic brachial plexopathies can be due to either compression or infiltration by localised pathologies, or a more diffuse or systemic cause. More common causes are neoplasia and radiation fibrosis. ${ }^{(9)}$ Other causes include neurogenic thoracic outlet syndrome, idiopathic brachial plexopathy (also known as neuralgic amyotrophy or Parsonage-Turner syndrome) and hereditary brachial plexopathy.

\section{Neoplastic brachial plexopathy}

Neoplastic brachial plexopathies may be due to primary tumours of the brachial plexus or metastatic spread from other cancers. Thus, the clinical history of a known primary malignancy is crucial in differentiating the causes of the plexopathy. Neoplastic brachial plexopathies tend to present with severe pain and weakness of the affected shoulder and upper limb. Physical examination commonly reveals involvement of the lower trunk, i.e. wasting of the small muscles of the hand, and loss of sensation in the C8 and T1 dermatomes. This is especially so in patients with Pancoast tumours, who may also have Horner's syndrome on examination.

Primary tumours of the brachial plexus, such as schwannomas and neurofibromas, tend to be benign and can occur in the setting of a systemic disease (e.g. neurofibromatosis type 1). Malignant primary tumours (e.g. neurofibrosarcoma), are less common. In the context of neurofibromatosis type 1, the multiple nerve lesions suggest peripheral neurofibromas. However, solitary neurofibromas can be difficult to distinguish from schwannomas on MRI. Both tend to show lesions arising from the nerve with T1-isointense and T2-hyperintense signal changes. ${ }^{(9)}$ The 'target sign' on T2-weighted images, seen as increased signal peripherally and decreased signal centrally, may be more commonly associated with neurofibromas. In contrast, the 'fascicular sign' seen on T2-weighted images, with rings of peripheral hyperintensity within the nerve, may suggest a schwannoma. ${ }^{(7)}$ Malignant primary tumours may show similar imaging features to benign tumours, which makes distinguishing them difficult. Features that may indicate malignancy include large size, heterogeneous appearance and irregular borders. ${ }^{(9)}$

Metastases from other sites are significantly more prevalent than primary tumours, with breast and lung carcinomas among the more common primary sites.$^{(7)}$ Breast carcinoma causes brachial plexopathy via metastasis to the axilla, while lung carcinomas may infiltrate the brachial plexus in cases of apical Pancoast tumours. Head and neck tumours can also affect the brachial plexus through both direct infiltration and metastatic spread. On MRI, neoplastic brachial plexopathy due to metastasis is seen as infiltration of the brachial plexus by masses. The masses usually appear hypointense on T1-weighted images and hyperintense on T2-weighted images, and show similar imaging characteristics to the primary lesion (Fig. 8). Lymphoma of the brachial plexus (peripheral neurolymphomatosis) is less common, and can occur in isolation or as part of a systemic lymphoma. Peripheral neurolymphomatosis affecting the brachial plexus appears on MRI as thickening of the nerves, with hypointensity on T1-weighted images and hyperintensity on T2-weighted images. Postcontrast enhancement may be seen $^{(7)}$ (Fig. 9).

\section{Radiation-induced brachial plexopathy}

Radiation-induced brachial plexopathy is caused by chronic inflammation of the affected nerves following radiotherapy for the treatment of cancer, eventually leading to fibrosis. Its incidence has declined to less than $1 \%$ today compared to many decades ago, and the risk is reduced with smaller total doses of radiotherapy. ${ }^{(10)}$ Radiotherapy for lung and breast carcinomas and lymphoma is the most common cause. The time interval between radiotherapy and the presentation of brachial plexopathy can range from months to decades. In contrast to neoplastic brachial plexopathy, radiationinduced plexopathy tends to cause weakness, numbness and paraesthesia of the affected limb, rather than pain. On MRI, features of radiation-induced brachial plexopathy ${ }^{(7,9)}$ include: (a) thickening of the nerves that appears diffuse, without a focal lesion; (b) contrast enhancement after gadolinium administration; and (c) T2-weighted images may show either 

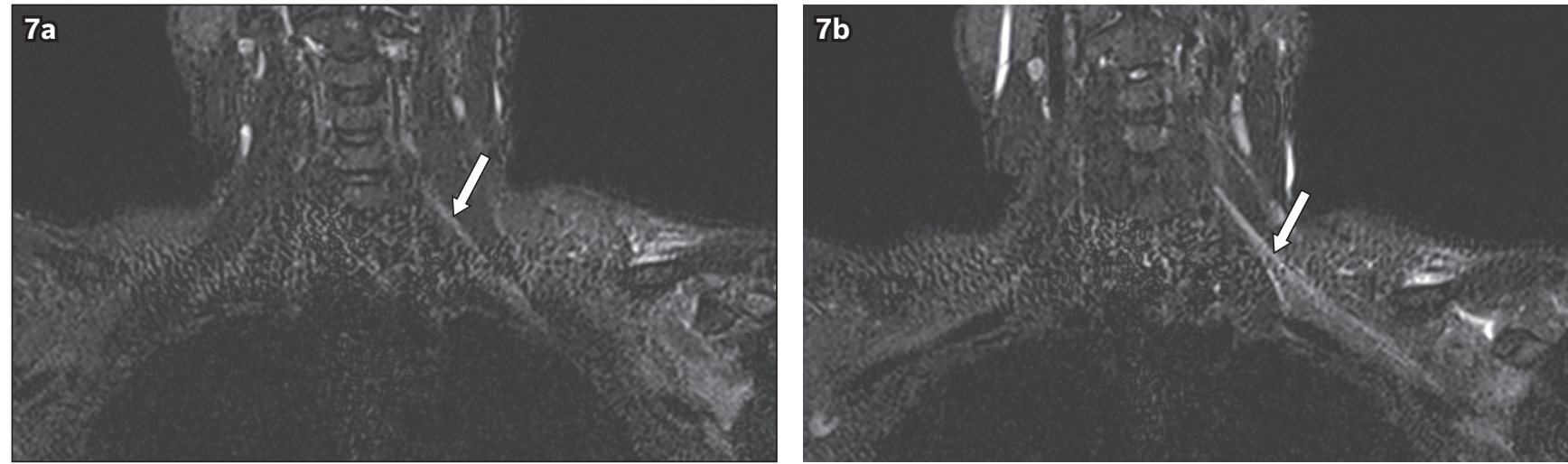

Fig. 7 A 36-year-old male construction worker sustained a left shoulder injury when a heavy object fell onto him. He presented with left upper limb pain and clinical signs of a left upper trunk brachial plexus injury. On MRI, coronal short tau inversion recovery sequence images show diffuse hyperintensity (arrows) of the (a) left C5 and C6 nerve roots, extending to the (b) trunks, divisions and cords. No disruption of the brachial plexus is evident. Given the presenting history, this is in keeping with a stinger injury of the left brachial plexus involving the $\mathrm{C} 5$ and $\mathrm{C} 6$ nerve roots.
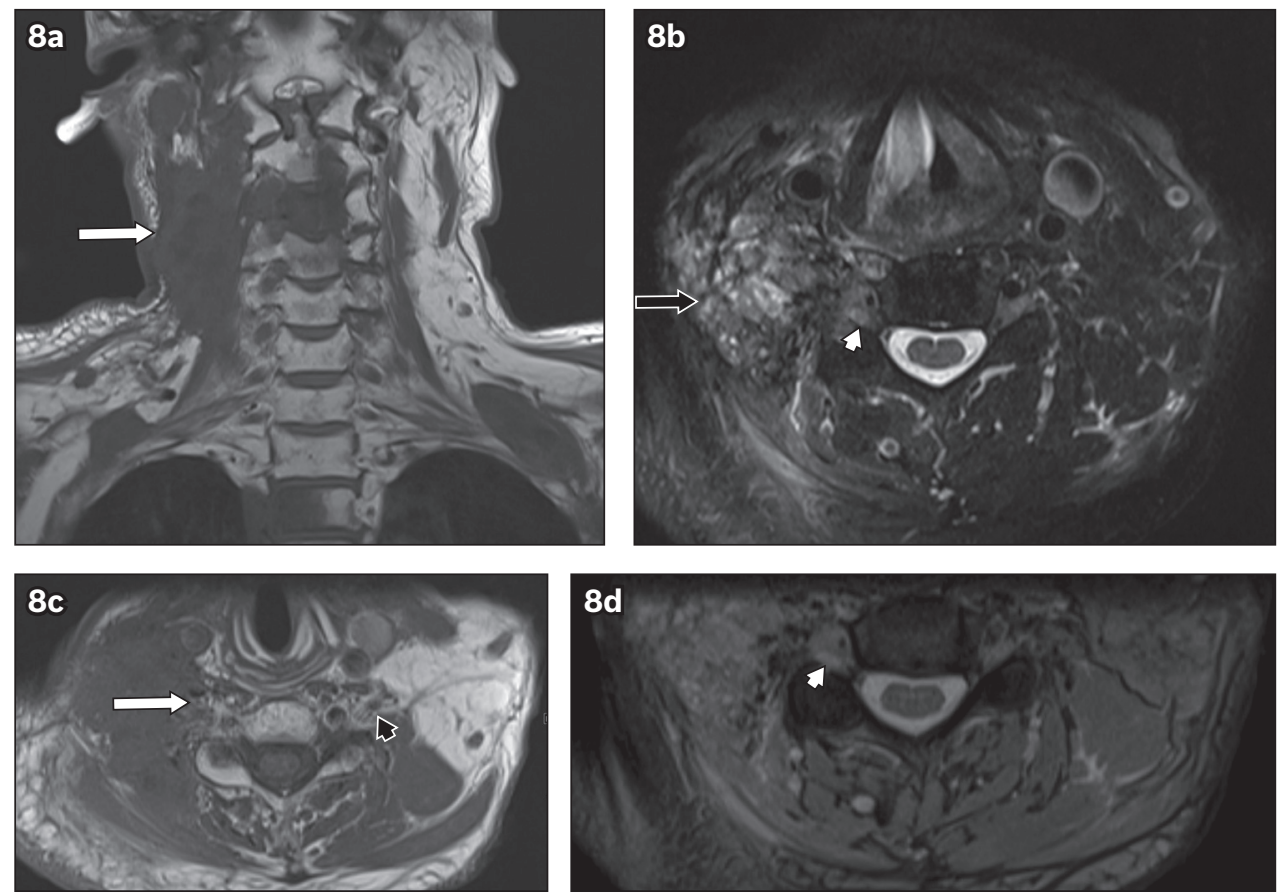

Fig. 8 A 60-year-old man with a history of nasopharyngeal carcinoma, previously treated with radiotherapy, presented with right neck pain and right upper limb weakness. On MRI, an infiltrative mass posterior to the carotid vessels on the right side of the neck was seen, which encased the exiting C3 to C5 nerve roots. (a) Coronal T1-W sequence image shows hypointensity (white arrow) and (b) axial T2-W sequence image shows heterogeneous hyperintensity (black arrow). (c) Axial T1-W image shows infiltration of the right C5 nerve root (white arrow) and an unaffected left C5 nerve root, in comparison (black arrowhead). Axial T2-W images show soft-tissue infiltration causing expansion of the right C5 neural foramen (white arrowheads in b \& d). Given the history of nasopharyngeal carcinoma, these findings are consistent with malignant infiltration of the right brachial plexus, which was confirmed on histology. T1-hypointense lesions (in a) in C3, C4 and T2 vertebrae are compatible with bony metastases. Normal flow signal in the right internal jugular vein is not seen, suggestive of compression (in $b \& c$ )

hyper- or hypointense signal changes (Fig. 10). However, it can often be difficult to distinguish plexopathy due to tumour recurrence from radiation-induced plexopathy.

\section{Neurogenic thoracic outlet syndrome}

True or classic neurogenic thoracic outlet syndrome is rare. It can be caused by congenital anomalies in the region of the brachial plexus, such as a fibrous band or a cervical rib, or compression between the anterior scalene muscle and the clavicle. The brachial plexus undergoes traction as it passes over the anomaly, leading to a plexopathy primarily involving the lower trunk (C8 and T1). ${ }^{(11)}$ Patients present with pain, weakness and numbness. Examination reveals wasting of the small muscles of the hand, and loss of sensation in the C8 and T1 dermatomes.

The degree of thoracic outlet obstruction is increased significantly when the arm is hyperabducted, as this causes reduction in the costoclavicular and interscalene spaces. MRI is thus usually performed with the arm by the side and subsequently hyperabducted. Features suggestive of thoracic outlet syndrome include: (a) narrowing of the costoclavicular space with hyperabduction of the arm; (b) loss of the fat plane around the brachial plexus; and (c) the brachial plexus being closely related to the adjacent bone. ${ }^{(12}$ 

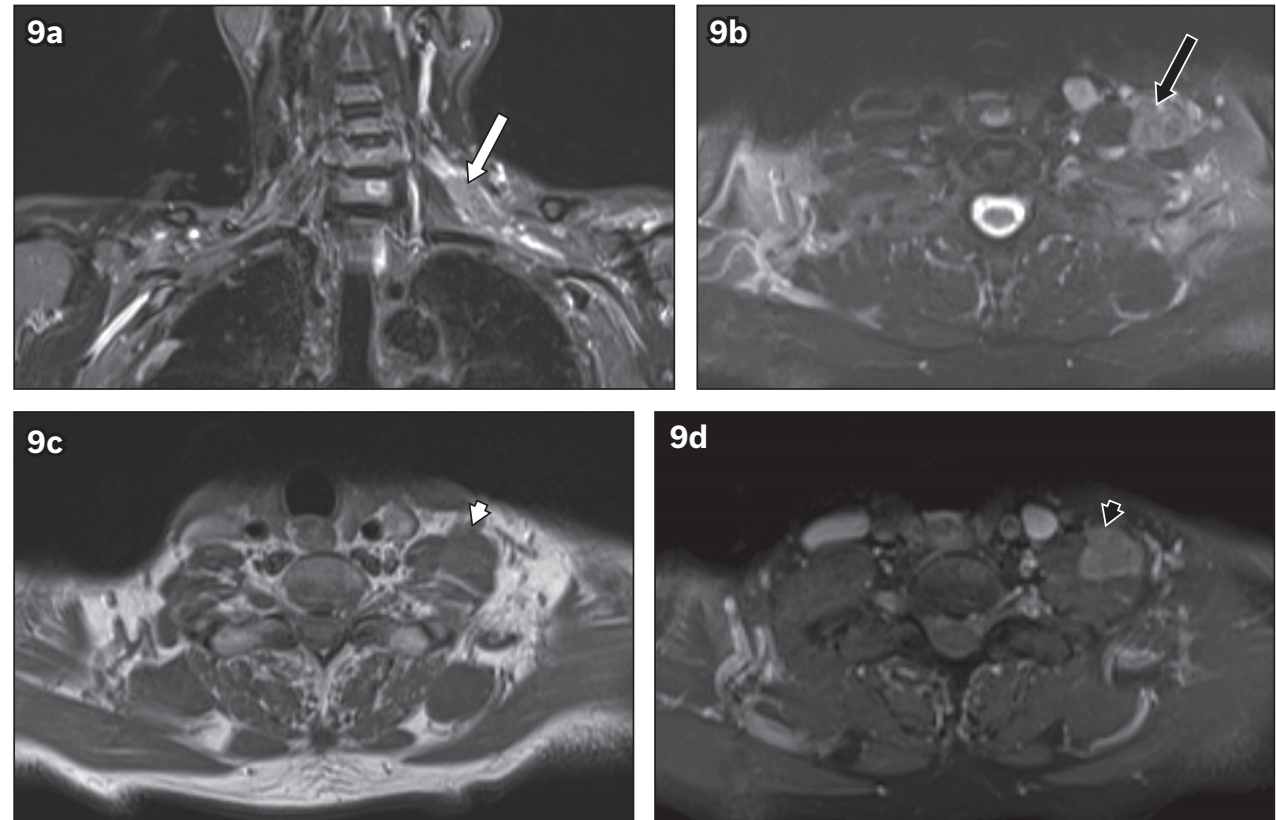

Fig. 9 A 74-year-old man presented with left upper limb pain and weakness of two months. Electromyography showed evidence of left upper trunk brachial plexopathy. On MRI, (a) coronal short tau inversion recovery sequence image shows a lobulated mass in the left supraclavicular region along the left C6 nerve root (white arrow). (b) T2-W image shows hyperintensity (black arrow) to muscle and (c) T1-W image shows isointensity (white arrowhead) to muscle. (d) Homogeneous contrast enhancement is seen (black arrowhead). These features suggest a neoplastic mass. Excision of the mass was performed and histology showed a diffuse large B-cell lymphoma. The patient subsequently underwent chemotherapy.
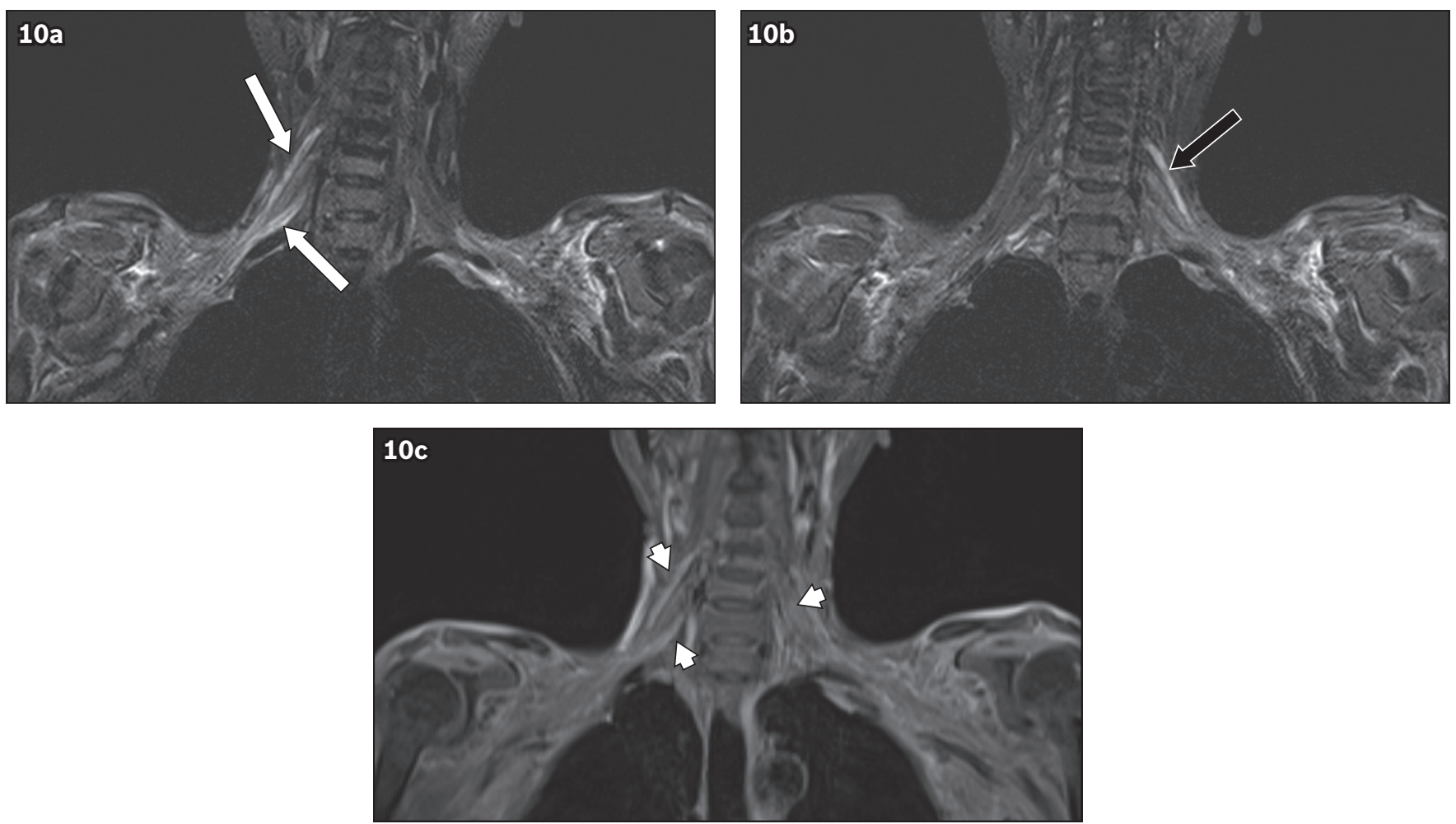

Fig. 10 A 52-year-old woman with a history of breast carcinoma, previously treated with radiotherapy, presented with right shoulder and arm pain, and bilateral hand numbness. On MRI, coronal short tau inversion recovery sequence image shows diffuse smooth thickening and T2 hyperintensity of the (a) right C5 and C7 nerve roots (white arrows) and the (b) left C5 nerve root (black arrow) to the level of the trunks, which show (c) diffuse contrast enhancement (arrowheads). Given the history of radiotherapy, these findings are in keeping with bilateral, radiation-induced brachial plexopathy.

\section{Idiopathic and hereditary brachial plexopathies}

Idiopathic brachial plexopathy, also known as neuralgic amyotrophy or Parsonage-Turner syndrome, is an inflammatory condition affecting the brachial plexus. The aetiology is unknown, although some pathologies are suspected, e.g. postviral or following vaccination. Patients present with acute onset of pain and numbness, and may develop weakness. MRI may appear normal in the first two weeks, after which intramuscular oedema manifests as T2 hyperintensity. Atrophy of the muscles later becomes apparent on MRI, reflecting the 
characteristic denervation injury; T1-hyperintense signal change also develops. The suprascapular nerve, and consequently the supraspinatus and infraspinatus muscles, are almost always involved. Importantly, structural abnormalities such as tumours will be absent. ${ }^{(13,14)}$ An autosomal dominant form of brachial plexopathy, associated with dysmorphism, has also been described. The symptoms of pain, numbness and weakness may present in childhood or adulthood, and may demonstrate a relapsing-remitting or progressive course.

\section{CONCLUSION}

Brachial plexopathy can result from many different aetiologies. MRI supplements clinical findings in aiding differentiation of the type of pathology. Accurate diagnosis has a large impact on the subsequent management. This series of cases illustrates some of the MRI findings for traumatic and non-traumatic brachial plexopathies.

\section{REFERENCES}

1. Chhabra A, Andreisek G, Soldatos T, et al. MR neurography: past, present, and future. AJR Am J Roentgenol 2011; 197:583-91.

2. Chhabra A, Williams EH, Wang KC, Dellon AL, Carrino JA. MR neurography of neuromas related to nerve injury and entrapment with surgical correlation. AJNR Am J Neuroradiol 2010; 31:1363-8.
3. Faglioni W Jr, Siqueira MG, Martins RS, Heise CO, Foroni L. The epidemiology of adult traumatic brachial plexus lesions in a large metropolis. Acta Neurochir (Wien) 2014; 156:1025-8.

4. Seddon HJ. A classification of nerve injuries. Br Med J 1942; 2:237-9.

5. Moran SL, Steinmann SP, Shin AY. Adult brachial plexus injuries: mechanism, patterns of injury, and physical diagnosis. Hand Clin 2005; 21:13-24.

6. Yoshikawa T, Hayashi N, Yamamoto S, et al. Brachial plexus injury: clinical manifestations, conventional imaging findings, and the latest imaging techniques. Radiographics 2006; 26 Suppl 1:S133-43.

7. Sureka J, Cherian RA, Alexander M, Thomas BP. MRI of brachial plexopathies. Clin Radiol 2009; 64:208-18.

8. Lawande M, Patkar DP, Pungavkar S. Pictorial essay: Role of magnetic resonance imaging in evaluation of brachial plexus pathologies. Indian J Radiol Imaging 2012; 22:344-9.

9. Wittenberg $\mathrm{KH}$, Adkins MC. MR imaging of nontraumatic brachial plexopathies: frequency and spectrum of findings. Radiographics 2000; 20:1023-32

10. Delanian S, Lefaix JL, Pradat PF. Radiation-induced neuropathy in cancer survivors. Radiother Oncol 2012; 105: 273-82.

11. Demondion $X$, Herbinet $P$, Van Sint Jan $S$, et al. Imaging assessment of thoracic outlet syndrome. Radiographics 2006; 26:1735-50.

12. Demondion X, Bacqueville E, Paul C, et al. Thoracic outlet: assessment with MR imaging in asymptomatic and symptomatic populations. Radiology 2003; 227:461-8.

13. Scalf RE, Wenger DE, Frick MA, Mandrekar JN, Adkins MC. MRI findings of 26 patients with Parsonage-Turner syndrome. AJR Am J Roentgenol 2007; 189:W39-44.

14. Gaskin CM, Helms CA. Parsonage-Turner syndrome: MR imaging findings and clinical information of 27 patients. Radiology 2006; 240:501-7. 


\section{SINGAPORE MEDICAL COUNCIL CATEGORY 3B CME PROGRAMME} (Code SMJ 201610B)

Question 1. Regarding magnetic resonance imaging (MRI) of the brachial plexus:

(a) Disadvantages of the two-dimensional (2D) short tau inversion recovery (STIR) sequence include suboptimal spatial resolution, occurrence of pulsation artefacts, and poor discrimination between nerves and blood vessels.

(b) A STIR sequence is superior to a 2D T2-weighted spectrally adiabatic inversion recovery (SPAIR) sequence on 3T scanners as it has a better signal-to-noise ratio and demonstrates more uniform fat suppression in larger fields of view.

(c) Three-dimensional STIR sampling perfection with application-optimised contrasts using different flip angle evolution sequences allow multiplanar reconstructions along the longitudinal axis of a nerve, which can better demonstrate pathological changes in a nerve.

(d) Intravenous gadolinium-based contrast is routinely used in imaging of the brachial plexus.

Question 2. Regarding Seddon's classification of nerve injuries:

(a) From least to most severe, the grades of nerve injury were termed neurotmesis, axonotmesis and neuropraxia.

(b) Wallerian degeneration occurs in neuropraxia and axonotmesis.

(c) In neuropraxia, conduction defects are temporary, with spontaneous and complete recovery.

(d) Spontaneous recovery is unlikely in neurotmesis and therefore it typically necessitates surgical repair.

Question 3. Regarding root avulsion injuries and preganglionic injuries of the brachial plexus:

(a) Root avulsion injuries due to traction of the nerve roots most commonly affect the $\mathrm{C} 5$ to $\mathrm{C} 7$ nerve roots.

(b) Pseudomeningocoeles form when the epidural sleeve is pulled away from the spinal cord.

(c) On MRI, oedema of the adjacent spinal cord, with hyperintense signal on T2-weighted images, is a feature of root avulsion injuries.

(d) Susceptibility artefacts on haeme-sensitive sequences, reflecting haemorrhage in the nerve root or adjacent spinal cord, is a feature of root avulsion injuries.

Question 4. Regarding postganglionic injuries of the brachial plexus:

(a) The majority of postganglionic injuries affect the roots and trunks.

(b) In the chronic phase of postganglionic rupture, atrophy of the supraspinatus and infraspinatus muscles may be seen.

(c) The continuity of the nerve is lost in neuropraxic postganglionic injury.

(d) Postganglionic nerve injuries usually have a better chance of functional recovery than preganglionic nerve injuries.

Question 5. Regarding neoplastic and radiation-induced brachial plexopathies:

(a) Primary tumours of the brachial plexus are more prevalent than metastatic spread from other sites.

(b) On T2-weighted imaging, the 'target sign' is associated with schwannomas, while the 'fascicular sign' is associated with neurofibromas.

(c) Imaging features that favour benign lesions include large size, heterogeneous appearance and irregular borders.

(d) Radiation-induced brachial plexopathy can occur decades after treatment with radiotherapy.

True

False

$\square$

$\square$

$\square$

$\square$
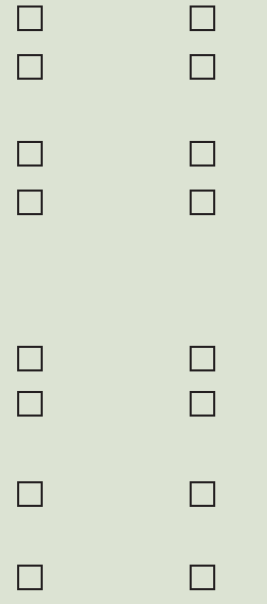

\section{Doctor's particulars:}

Name in full

MCR number

Specialty:

Email address

\section{SUBMISSION INSTRUCTIONS:}

(1) Visit the SMJ website: http://www.smj.org.sg/current-issue and select the appropriate set of questions. (2) Provide your name, email address and MCR number. (3) Select your answers and click "Submit".

RESULTS:

(1) Answers will be published online in the SMJ December 2016 issue. (2) The MCR numbers of successful candidates will be posted online at the SMJ website by 5 December 2016. (3) Passing mark is $60 \%$. No mark will be deducted for incorrect answers. (4) The SMJ editorial office will submit the list of successful candidates to the Singapore Medical Council. (5) One CME point is awarded for successful candidates.

Deadline for submission: (October 2016 SMJ 3B CME programme): 12 noon, 28 November 2016. 